\title{
Downregulation of VEGF and upregulation of TL1A expression induce HUVEC apoptosis in response to high glucose stimuli
}

\author{
MIAO YU ${ }^{1,2}$, GUIHUA LU ${ }^{1,2}$, XUN ZHU $^{3,4}$, ZHIBIN HUANG $^{1}$, CHONG FENG $^{1}$, \\ RONG FANG $^{2}$, YESONG WANG ${ }^{1}$ and XIUREN GAO ${ }^{1}$ \\ ${ }^{1}$ Department of Cardiology, First Affiliated Hospital of Sun Yat-sen University, Guangzhou, Guangdong 510080; \\ ${ }^{2}$ Key Laboratory of Assisted Circulation, Ministry of Health, Guangzhou, Guangdong 710068; \\ ${ }^{3}$ Department of Microbiology, Zhongshan School of Medicine, Sun Yat-sen University, Guangzhou, Guangdong 510080; \\ ${ }^{4}$ Key Laboratory of Tropical Disease Control, Ministry of Education, Guangzhou, Guangdong 510275, P.R. China
}

Received March 3, 2015; Accepted December 30, 2015

DOI: $10.3892 / \mathrm{mmr} .2016 .4924$

\begin{abstract}
High glucose-induced endothelial cell apoptosis is considered to be the initiator of diabetes-associated vascular complications. Experiments in vivo and in vitro have demonstrated that high glucose levels contribute to the apoptosis of endothelial cells by mediating cellular dysfunction and metabolic disorder via the production of various cytokines. As the most important endogenous vascular regulators, the balance between pro-proliferative effector vascular endothelial growth factor (VEGF) and anti-proliferative effector tumor necrosis factor-like cytokine 1A (TL1A) is important in the modulation of endothelial cell survival and proliferation, and neovascularization. The present study aimed to explore whether the imbalance between VEGF and TL1A affected the apoptosis of human umbilical vein endothelial cells (HUVECs) exposed to high glucose conditions and then further investigated the potential mechanism. The results showed that the downregulation of VEGF in combination with the upregulation of TL1A in response to high glucose levels led to enhanced HUVEC apoptosis. Further experiments revealed that silencing high glucose-induced TL1A expression using TL1A small interfering (si)RNA or the overexpression of VEGF by transfection with VEGF DNA resulted in a reduced HUVEC apoptosis rate compared with the controls. The effects occurred by attenuating and activating the phosphoinositide 3-kinase/Akt/endothelial nitric oxide synthase pathway, respectively. In addition, VEGF and TL1A inhibited each other in hyperglycemia. In conclusion, these findings provide theoretical support for the further
\end{abstract}

Correspondence to: Professor Yesong Wang or Professor Xiuren Gao, Department of Cardiology, First Affiliated Hospital of Sun Yat-sen University, 58 Zhongshan Road, Guangzhou, Guangdong 510080, P.R. China

E-mail: yesong_wang@aliyun.com

E-mail: xiurengao@163.com

Key words: high glucose, vascular endothelial growth factor, tumor necrosis factor-like cytokine $1 \mathrm{~A}$, apoptosis investigation of novel therapeutic strategies designed to maintain the balance between VEGF and TL1A and, thus, to prevent the onset and progression of endothelial cell apoptosis in response to high glucose stimuli.

\section{Introduction}

As a metabolic disorder, diabetes has been defined as an independent risk factor for various types of cardiovascular disease. It has been recognized that endothelial cell dysfunction and apoptosis represent the beginning of diabetes-associated vascular disease $(1,2)$. The maintenance of the balance between the proliferation, apoptosis and necrosis of endothelial cells is multi-factorial and involves the interaction between vascular endothelial growth factor (VEGF) and tumor necrosis factor-like cytokine 1A (TL1A).

VEGF, considered as the most important cytokine in promoting endothelial cell growth, exhibits a crucial role in maintaining the stability of vascular endothelial structure and function by stimulating endothelial cell proliferation and regeneration while restraining apoptosis. Elevated VEGF levels are observed as a cardiac response under conditions of ischemia-hypoxia, while impairments in VEGF expression and activity have been identified in patients with diabetes (3-5). It has been reported that patients without diabetes exhibited increased VEGF expression in response to myocardial ischemia and infarction but that diabetic patients exhibited reduced VEGF levels in the myocardium and in chronic wounds $(6,7)$. The downregulation of VEGF expressed in tissues, aside from the kidney and the retina, contributes to the decrease in ischemia-induced collateral vessel formation in patients with diabetes, resulting in necrosis and irreversible damage (3). Thus, VEGF is a key component in the mechanism that regulates endothelial cell proliferation and neovascularization.

TL1A is a unique endogenous inhibitor of angiogenesis $(8,9)$ that is predominantly produced by endothelial cells and that performs its physiological functions by binding to its receptors, DR3 and DcR3 (10). As a multifunctional member of the tumor necrosis factor super-family, TL1A participates in numerous physiological processes, such as the immune response (11), and in pathologies such as inflammatory bowel 
disease (12) and atherosclerosis $(13,14)$. It has been widely accepted that TL1A exerts an inhibitory effect on endothelial cell proliferation during different growth stages by promoting apoptosis or growth arrest (15). Previous experiments revealed that senescent human umbilical vein endothelial cells (HUVECs) displayed enhanced TL1A expression (16). Additionally, TL1A inhibits tumor angiogenesis and tumor growth, and these effects are consistent with the observation that TL1A expression is significantly decreased in various types of cancer, such as ovarian (17) and breast cancer (18), as well as in wound tissue (19). Thus, TL1A functions as a suppressor of neovascularization and endothelial cell proliferation.

As important components of the mechanisms regulating neovascularization and cell proliferation, the balance between TL1A and VEGF ensures the stability of the established vasculature. The present study investigated TL1A and VEGF expression in high glucose-induced apoptotic HUVECs, with the aim of elucidating the mechanism by which TL1A and VEGF act on HUVECs. The experiments aimed to provide a novel strategy to protect endothelial cells from high glucose-induced apoptosis.

\section{Materials and methods}

Chemicals and reagents. Anti-glyceraldehyde 3-phosphate dehydrogenase (GAPDH) mouse monoclonal antibody (mAb; HRP-60004) and the horseradish peroxidase (HRP)-conjugated Affinipure goat anti-mouse (SA00001-1) and anti-rabbit (SA00001-2) secondary immunoglobulin $(\mathrm{Ig}) \mathrm{G}(\mathrm{H}+\mathrm{L})$ antibodies were purchased from Proteintech Group (Wuhan, China). Anti-phospho-phosphoinositide 3-kinase (PI3K) p85 (Tyr458)/p55 (Tyr199) rabbit polyclonal antibody (pAb; 4228), the anti-PI3K p85 rabbit pAb (4257), the anti-phospho-Akt (Ser473) rabbit $\mathrm{mAb}$ (4060), the anti-Akt rabbit $\mathrm{mAb}$ (4691), the anti-phospho-endothelial nitric oxide synthase (eNOS) (Ser1177) rabbit pAb (9571), and the anti-eNOS rabbit pAb (9572) were purchased from Cell Signaling Technologies Inc. (Beverly, MA, USA). Anti-VEGF mouse pAb (ab46154) and anti-TL1A rabbit pAb (ab21272) were purchased from Abcam (Cambridge, UK). Endothelial cell medium (ECM) was purchased from ScienCell Research Laboratories (San Diego, CA, USA).

Cell culture. The HUVECs used in this study were purchased from ScienCell Research Laboratories. The cells were cultured in $\mathrm{ECM}$ at $37^{\circ} \mathrm{C}$ in a humidified incubator containing $5 \% \mathrm{CO}_{2}$. Cultured endothelial cells of 3-5 passages were used for the following experiments. HUVECs were cultured in conditioned medium with various concentrations of glucose $(5.5,15$ and $33 \mathrm{mM} / \mathrm{l})$ for various time periods $(0,24$ and $48 \mathrm{~h})$. Furthermore, HUVECs were transfected with TL1A siRNA, negative control siRNA or VEGF DNA. Following transfection for $12 \mathrm{~h}$, cells were cultured in conditioned medium with $5.5 \mathrm{mM} / 1 \mathrm{NG}$ or $33 \mathrm{mM} / \mathrm{l} \mathrm{HG}$ for 0,24 or $48 \mathrm{~h}$.

Western blot analysis. Western blot analysis was performed as follows: Total proteins were extracted from cells cultured in a 6 -well plate $\left(3 \times 10^{5}\right.$ cells/well $)$ in triplicate using radioimmunoprecipitation assay buffer (Invitrogen; Thermo Fisher Scientific Inc., Waltham, MA, USA). After centrifugation at $16,100 \times \mathrm{g}$ at $4^{\circ} \mathrm{C}$, the supernatant was collected, and the protein concentration was quantified using a bicinchoninic acid protein assay kit (Pierce, Rockford, IL, USA). Then, $30 \mu \mathrm{g}$ of each total protein extract was separated via $8 \%$ sodium dodecyl sulfate-polyacrylamide gel electrophoresis gels (Amresco, LLC, Solon, OH, USA) and transferred to a polyvinylidene difluoride membrane (Roche Diagnostics, Indianapolis, IN, USA). The membranes were blocked in $5 \%$ milk or $5 \%$ bovine serum albumin (Invitrogen; Thermo Fisher Scientific, Inc.), depending on the level of background, and then probed with the following primary antibodies overnight at $4^{\circ} \mathrm{C}$ : Mouse anti-VEGF (1:500) pAb and rabbit anti-phospho-phosphoinositide 3-kinase (PI3K) p85 (Tyr458)/p55 (Tyr199) pAb, anti-PI3K p85, anti-phospho-Akt (Ser473), anti-Akt, anti-phospho-endothelial nitric oxide synthase (eNOS) (Ser1177), anti-eNOS (all 1:1,000) and anti-TL1A (1:500) and mouse anti-GAPDH $(1: 10,000)$. The following day, the membranes were washed three times with $1 \mathrm{X}$ Tris-buffered saline with Tween-20 and incubated in HRP-conjugated Affinipure goat anti-mouse $(1: 1,000)$ and anti-rabbit $(1: 2,000)$ secondary $\operatorname{IgG}(\mathrm{H}+\mathrm{L})$ antibodies secondary antibodies for $1 \mathrm{~h}$ at room temperature. Protein signals were visualized using enhanced chemiluminescence (Millipore, Boston, MA, USA), and the gray analysis of bands were relatively quantified using Quantity One software (version 4.62; Bio-Rad Laboratories, Hercules, CA, USA).

Reverse transcription-quantitative polymerase chain reaction $(R T-q P C R)$. Total RNA was extracted from HUVECs using TRIzol reagent (Sigma-Aldrich, St. Louis, MO, USA) according to the manufacturer's instructions, and the RNA concentrations were measured using a NanoDrop 2000 (Thermo Fisher Scientific Inc.). RNA was reverse transcribed as follows: $4 \mu \mathrm{l}$ sample RNA $(0.5 \mu \mathrm{g} / \mu \mathrm{l}), 1 \mu \mathrm{l}$ random primer $\left(25 \mu \mathrm{M}\right.$ ) and $1 \mu \mathrm{l}$ RNase-free $\mathrm{dH}_{2} \mathrm{O}$ (both Bio-Rad Laboratories, Inc.) were mixed together in a $0.2 \mathrm{ml}$ Eppendorf tube, incubated at $70^{\circ} \mathrm{C}$ for $10 \mathrm{~min}$ and immediately cooled on ice for $\geq 2 \mathrm{~min}$. Following centrifugation at 3,000 $\mathrm{x} \mathrm{g}$ for several seconds, $0.25 \mu \mathrm{l}$ RTase M-MLV, $2 \mu \mathrm{l}$ M-MLV buffer (5X), $0.5 \mu \mathrm{l}$ dNTPs (2.5 mM each), $0.25 \mu 1$ RNase inhibitor (40 u/ $\mu \mathrm{l}$; all Bio-Rad Laboratories, Inc.) and $1 \mu \mathrm{l}$ RNase-free $\mathrm{dH}_{2} \mathrm{O}$ was added and incubated at $30^{\circ} \mathrm{C}$ for $10 \mathrm{~min}$, followed by $1 \mathrm{~h}$ at $42^{\circ} \mathrm{C}$ and $15 \mathrm{~min}$ at $70^{\circ} \mathrm{C}$ prior to cooling on ice. qPCR for VEGF and TL1A was performed using a qPCR kit (SYBR Green; Roche, Ltd., Basel, Switzerland). The primer sequences used were as follows: Forward: 5'-TCTCTACCC CAGGTCAGACG-3' and reverse: 5'-TCCCCAAACTCCTGG TCAGA-3' for VEGF; forward: 5'-TGTGCAGTTCCAGGC TCTAAAA-3' and reverse: 5'-CCGTCTGCTCTAAGAGGT GCAT-3' for TL1A; and forward: 5'-GGTGGTCTCCTCTGA CTTCAACA-3' and reverse: 5'-GTTGCTGTAGCCAAATTC GTTGT-3' for GAPDH (Invitrogen; Thermo Fisher Scientific Inc.). The reverse transcription conditions were as follows: $95^{\circ} \mathrm{C}$ for $30 \mathrm{sec}$ for pre-incubation, followed by 45 cycles of $50^{\circ} \mathrm{C}$ for $40 \mathrm{sec}$ and $72^{\circ} \mathrm{C}$ for $40 \mathrm{sec}$. Transcript amplification and detection were performed using a LightCycler480 thermal cycler (Roche Diagnostics GmbH, Manheim, Germany) as follows: Pre-incubation for $5 \mathrm{~min}$ at $95^{\circ} \mathrm{C}$, amplification via 
A

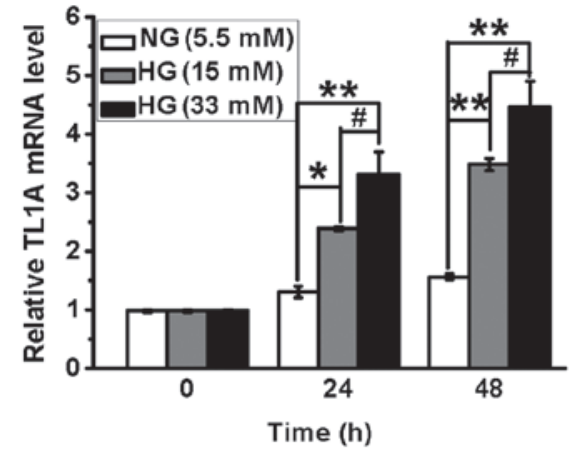

C

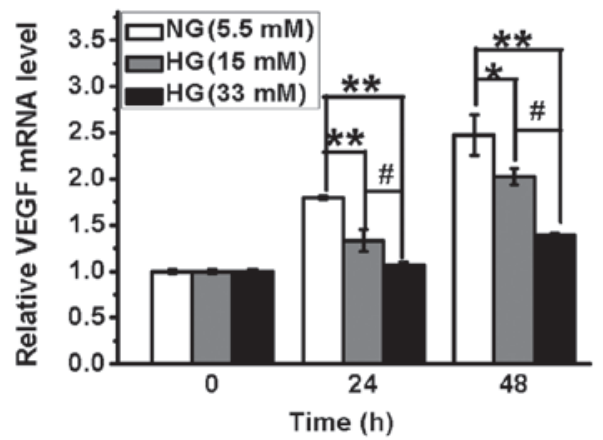

B

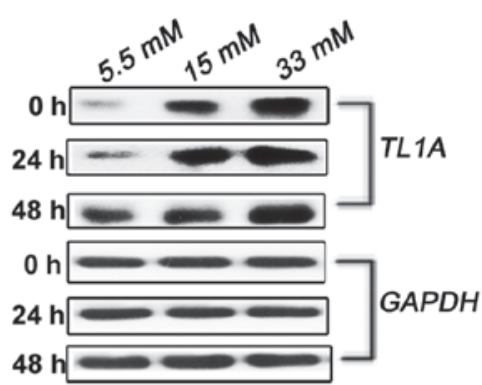

D

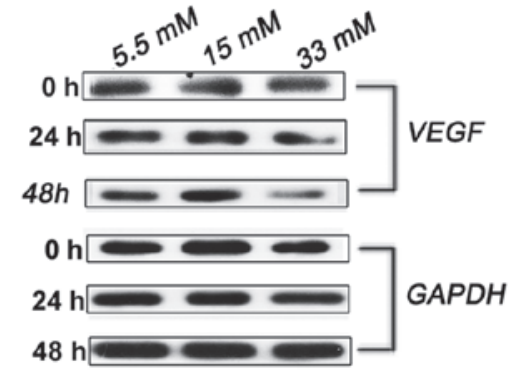

Figure 1. High glucose levels result in upregulation of TL1A expression and downregulation of VEGF expression in HUVECs. HUVECs were cultured in conditioned medium with different concentrations of glucose (5.5, 15 and $33 \mathrm{mM} / \mathrm{l})$. TL1A mRNA and protein levels were analyzed by (A) RT-qPCR and (B) western blot analysis at different time intervals, respectively. VEGF mRNA and protein levels were analyzed by (C) RT-qPCR and (D) western blot analysis at different time intervals, respectively. GAPDH served as a control. NG, normal glucose. HG, high glucose; HUVEC, human umbilical vein endothelial cell; TL1A, tumor necrosis factor-like cytokine 1A; VEGF, vascular endothelial growth factor; RT-qPCR, reverse transcription-quantitative polymerase chain reaction; GAPDH, glyceraldehyde 3-phosphate dehydrogenase. " $\mathrm{P}<0.05,{ }^{* *} \mathrm{P}<0.01$ vs. NG. $\mathrm{P}<0.05$ vs. HG.

45 cycles of $10 \mathrm{sec}$ at $95^{\circ} \mathrm{C}, 10 \mathrm{sec}$ at $60^{\circ} \mathrm{C}$ and $20 \mathrm{sec}$ at $72^{\circ} \mathrm{C}$, and a final cooling step of $30 \mathrm{sec}$ at $40^{\circ} \mathrm{C}$ (ramp rate of $\left.4.4^{\circ} \mathrm{C} / \mathrm{s}\right)$. The relative quantity of each gene was obtained using the $\Delta \Delta \mathrm{Cq}$ method (20).

Gene silencing. RNA interference was performed using TL1A small interfering (si)RNA and control siRNA (RiboBio, Guangzhou, China). Cell cultures at 40-60\% confluence were transfected with siRNA at a final concentration of $50 \mathrm{nM}$ using Lipofectamine 2000 (Invitrogen; Thermo Fisher Scientific Inc.) according to the manufacturer's instructions. After transfection (12 h), HUVECs were used for experimentation.

VEGF overexpression. The cells were seeded in 6-well plates ( $3 \times 10^{5}$ cells/well) and were incubated for $24 \mathrm{~h}$ prior to transfection. VEGF DNA was transfected into the HUVECs at a final concentration of $3 \mu \mathrm{g} / \mu \mathrm{l}$ using Lipofectamine 2000. Following transfection for 4-6 $\mathrm{h}$, the transfection medium was replaced with ECM and culturing was continued for $24 \mathrm{~h}$ prior to further treatment.

Apoptosis assay via flow cytometry (FCM). Apoptosis was measured using an Annexin V-fluorescein isothiocyanate (FITC) Apoptosis Assay kit (Nanjing KeyGen Biotech. Co. Ltd., Nanjing, China) according to the manufacturer's instructions. After transfection with TL1A siRNA or VEGF DNA, the HUVECs incubated in high glucose $(33 \mathrm{mM} / \mathrm{l})$ for various time periods $(0,24$ or $48 \mathrm{~h})$ were harvested and stained with Annexin V-FITC and propidium iodide (PI; 1:100 BD Biosciences; Franklin Lakes, NJ, USA) for $15 \mathrm{~min}$ at room temperature in the dark. Following incubation, flow cytometry (FACS Calbur; BD Biosciences) was performed to detect the apoptosis of HUVECs. The axes were set as Annexin V on the horizontal axis and PI on the vertical axis, in order to determine the baseline of the axes according to the blank control and distinguish normal cells from apoptotic cells. Four-quadrant were used; the left lower quadrant (Annexin $\mathrm{V}^{-}-\mathrm{PI}^{-}$) represented normal cells, the left upper quadrant (Annexin $\mathrm{V}^{-}-\mathrm{PI}^{+}$) represents fragmented or injured cells, the right lower quadrant $\left(\right.$ Annexin $\left.\mathrm{V}^{+}-\mathrm{PI}^{-}\right)$represented viable apoptotic cells, the right upper quadrant( Annexin $\mathrm{V}^{+}-\mathrm{PI}^{+}$) represented non-viable apoptotic or non-viable non-apoptotic cells. Both viable apoptotic and non-viable apoptotic cells or non-viable non-apoptotic cells were used to calculate cell apoptosis.

Statistical analysis. All experiments were performed at least three times and all data are expressed as the mean \pm standard deviation. Statistical analysis of the data was performed via one-way analysis of variance using SPSS 13.0 software (SPSS, Inc., Chicago, IL, USA). P $<0.05$ was considered to indicate a statistically significant difference.

\section{Results}

TL1A expression is induced in HUVECs in response to high glucose. To determine whether TL1A is induced in response to high glucose, HUVECs were treated with different concentrations of glucose $(5.5,15$ or $33 \mathrm{mM})$ for different periods $(0,24$ or $48 \mathrm{~h})$ and the changes in TL1A expression 
A

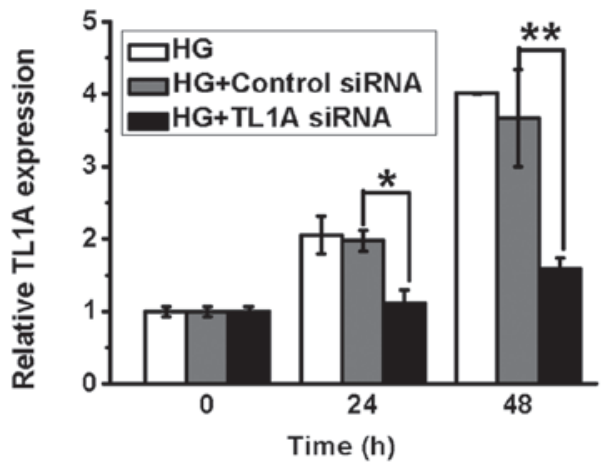

B

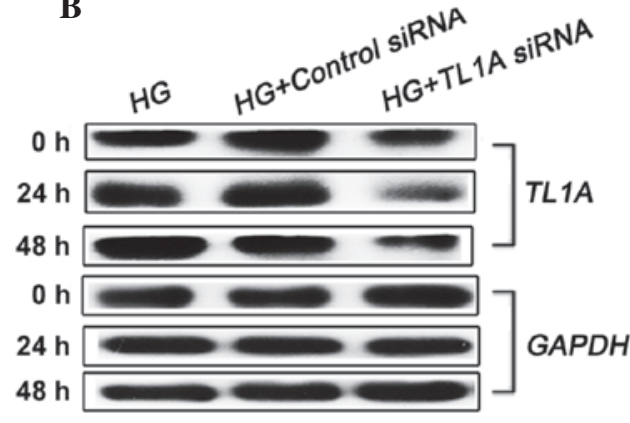

C

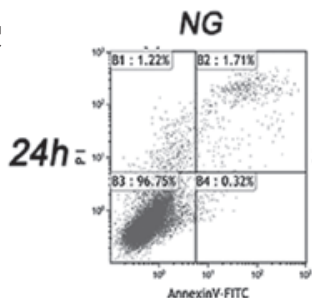

HG (33 mM) HG+Control siRNA HG+TL1A siRNA HG+VEGF DNA
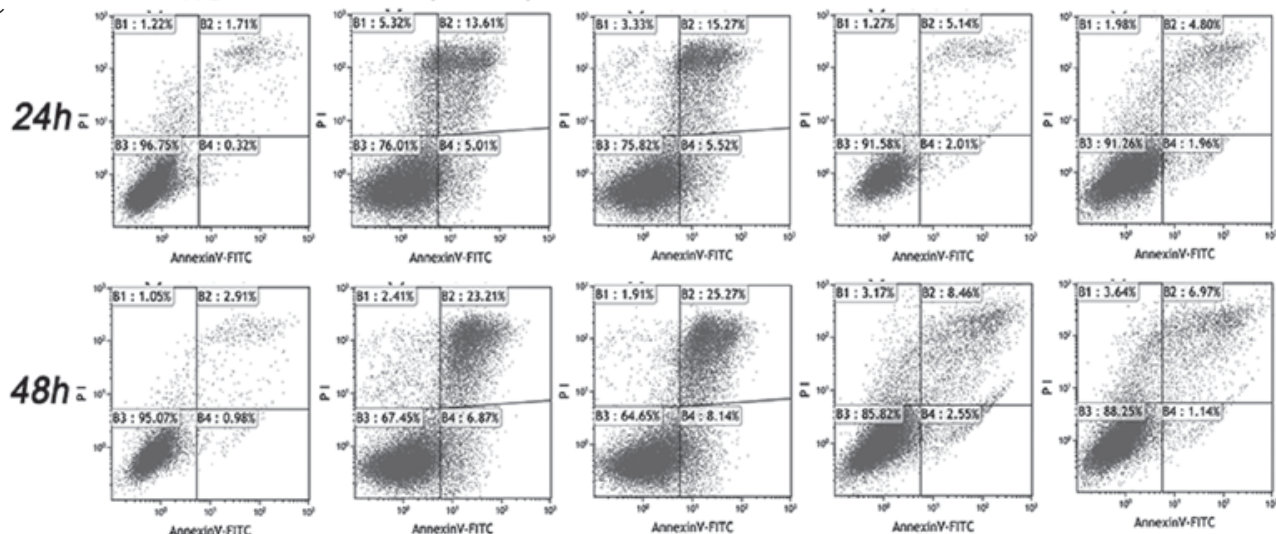

D

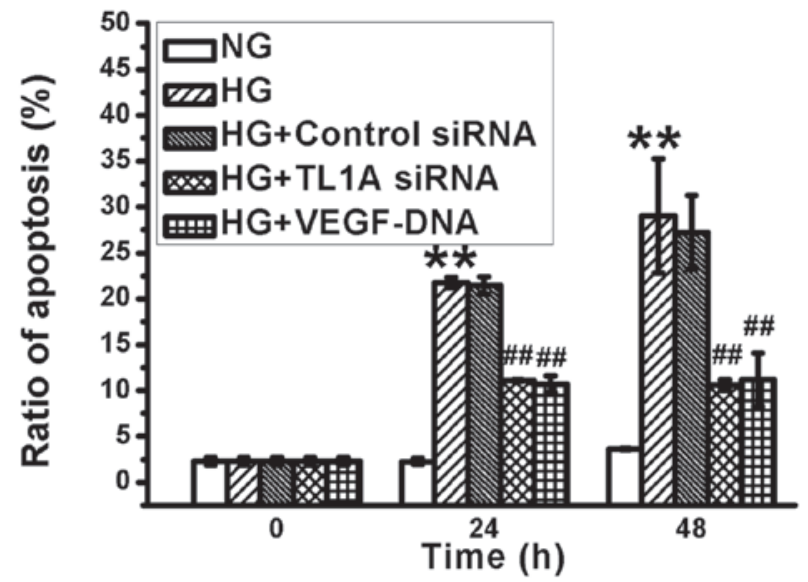

E
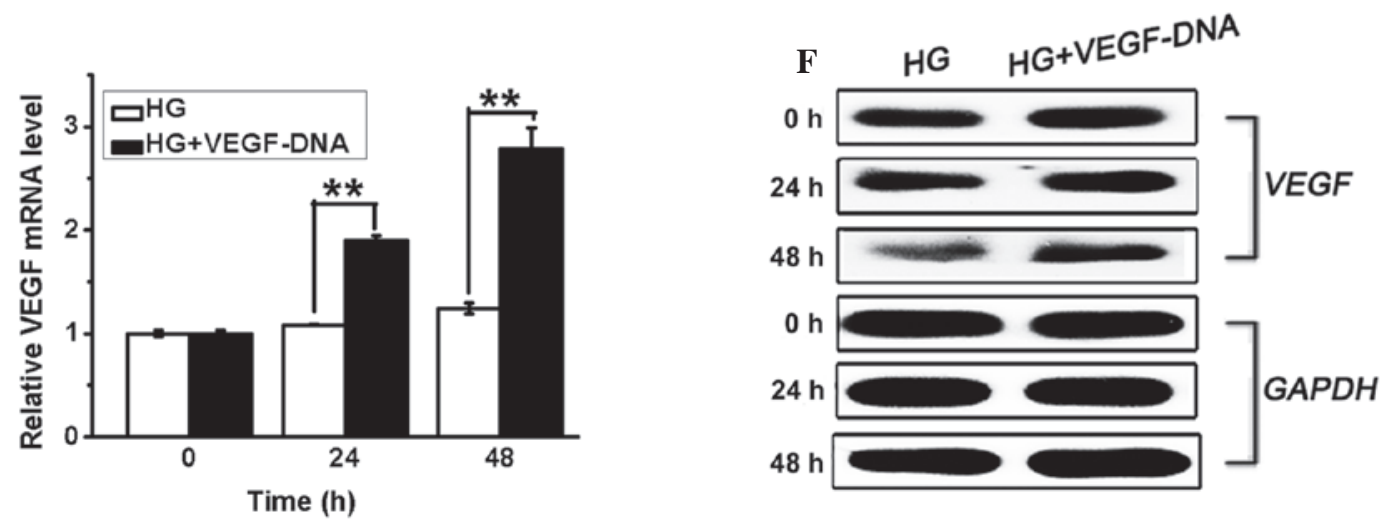

Figure 2. Silencing of HG-induced TL1A expression or overexpression of VEGF levels attenuates HUVEC apoptosis in response to high glucose. HUVECs were transfected with TL1A siRNA, negative control siRNA or VEGF DNA. After transfection for $12 \mathrm{~h}$, cells were cultured in conditioned medium with NG $(5.5 \mathrm{mM} / 1)$ or HG $(33 \mathrm{mM} / 1)$ for different times $(0,24$ or $48 \mathrm{~h})$. TL1A expression was detected at the (A) mRNA and (B) protein levels to verify the efficiency of TL1A siRNA transfection. (C and D) HG-induced apoptosis of HUVECs treated with or without TL1A siRNA or VEGF DNA were assessed by flow cytometry. VEGF expression at the (E) mRNA and (F) protein levels was detected to verify the efficiency of VEGF DNA transfection. NG, normal glucose; HG, high glucose; TL1A, tumor necrosis factor-like cytokine 1A; VEGF, vascular endothelial growth factor; siRNA, small interfering RNA; ${ }^{\mathrm{P}}<0.05$, ${ }^{* * *} \mathrm{P}<0.01$ vs. NG. ${ }^{\# \#} \mathrm{P}<0.01$ vs. $\mathrm{HG}$. 
A

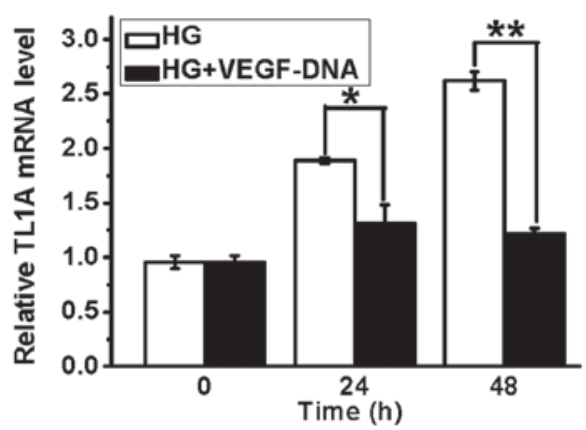

C

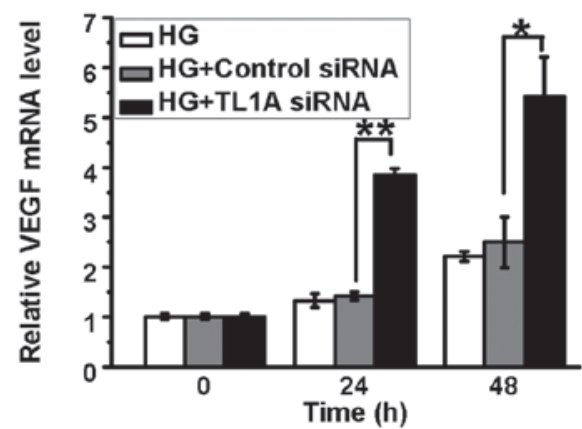

B

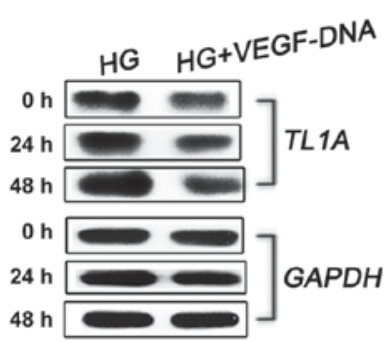

D

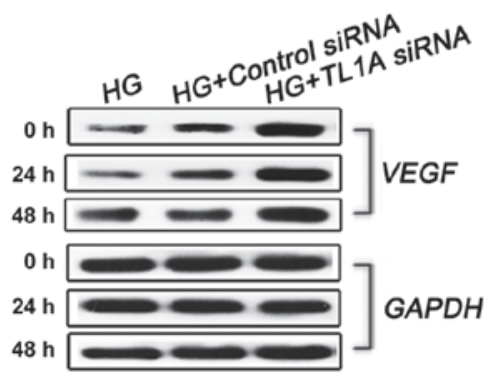

Figure 3. TL1A and VEGF exhibit an inhibitory effect on each other in high glucose conditions. (A) Analysis of TL1A mRNA in HUVECs cultured under HG medium in the absence or presence of VEGF DNA via RT-qPCR. (B) Western blot analysis of TL1A protein in HUVECs transfected with or without VEGF DNA. Levels of VEGF (C) mRNA and (D) protein determined at the indicated time points in HUVEC cultured under high glucose medium with the absence or presence of TL1A siRNA via RT-qPCR or western blot analysis, respectively. TL1A, tumor necrosis factor-like cytokine 1A; VEGF, vascular endothelial growth factor; HUVECs, human umbilical vein endothelial cells; RT-qPCR, reverse transcription quantitative polymerase chain reaction; siRNA, small interfering RNA; HG, high glucose; GAPDH, glyceraldehyde 3-phosphate dehydrogenase. ${ }^{*} \mathrm{P}<0.05,{ }^{* *} \mathrm{P}<0.01$ vs. HG.
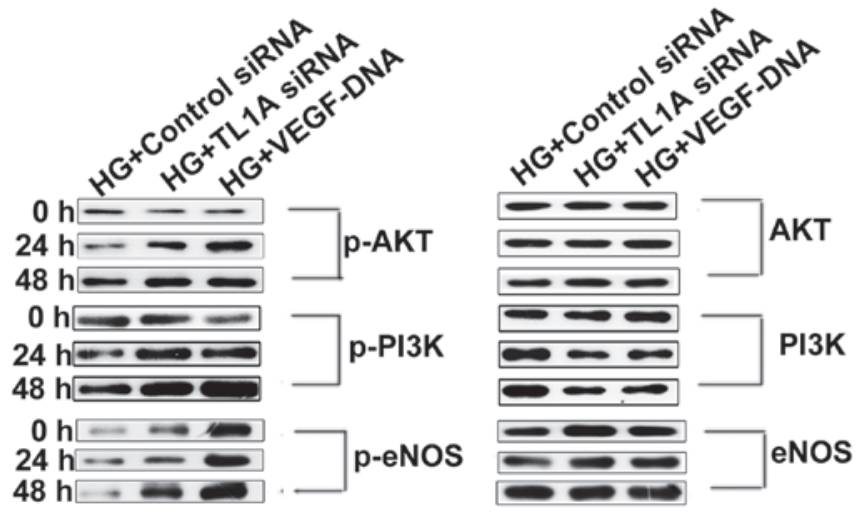

Figure 4. Elevated expression of TL1A and reduced expression of VEGF in response to high glucose attenuated the PI3K-Akt-eNOS signaling pathway. HUVECs were transfected with TL1A siRNA, negative control siRNA and VEGF DNA separately. After transfection for $12 \mathrm{~h}$, cells were cultured in conditioned medium with high glucose $(33 \mathrm{mM} / \mathrm{l})$ for indicated time points. Western blot analysis was used to determine the quantity of PI3K, Akt, eNOS and their phosphorylated forms. HG, high glucose; siRNA, small interfering RNA; TL1A, tumor necrosis factor-like cytokine 1A; VEGF, vascular endothelial growth factor; HUVECs, human umbilical vein endothelial cells; p-, phosphorylated; PI3K, phosphoinositide 3-kinase; eNOS, endothelial nitric oxide synthase.

were determined via RT-qPCR and western blot analysis. As shown in Fig. 1A and B, TL1A expression at the mRNA and protein levels was induced by high glucose, resulting in a significant increase compared with the controls at 24 and $48 \mathrm{~h}$. These results demonstrated that high glucose induces TL1A expression in HUVECs in a concentration-dependent manner.
VEGF expression is reduced in HUVECs in response to high glucose. Different VEGF-regulating mechanisms resulted in distinct levels of VEGF expression between retinal and cardiac tissue in response to high glucose (3). A previous study has shown that VEGF expression was increased by 2 -fold in the retina and in glomeruli in diabetes (3). To evaluate VEGF expression in HUVECs under hyperglycemic conditions, the HUVECs were treated as described above and the expression of VEGF was measured by RT-qPCR and western blot analysis. As shown in Fig. 1C and D, VEGF expression was decreased in the presence of high glucose compared with normal glucose. Thus, VEGF expression is inhibited in HUVECs cultured in the presence of high glucose.

Increase in TLIA expression and the decrease in VEGF expression promotes HUVEC apoptosis in response to high glucose. TL1A and VEGF expression levels in HUVECs displayed opposing changes in response to the high glucose stimulus, and it has previously been demonstrated that high glucose levels induce endothelial cell apoptosis (21). To determine the association between the changes in TL1A and VEGF expression and the induction of HUVEC apoptosis in hyperglycemia, HUVECs were transfected with VEGF DNA $(3 \mu \mathrm{g} / \mu \mathrm{l})$ or TL1A siRNA $(50 \mathrm{nM})$. Apoptosis of these cells was then evaluated via flow cytometry. As shown in Fig. 2A and B, TL1A siRNA effectively suppressed high glucose-induced TL1A expression at the mRNA and protein levels after stimulation in high glucose for 24 and $48 \mathrm{~h}$. Furthermore, the silencing of high glucose-induced TL1A expression significantly attenuated high glucose-induced cell apoptosis (Fig. 2C and D). Furthermore, the transfection of VEGF DNA, which 
increased the VEGF levels (Fig. 2E and F) in HUVECs, also protected against high glucose-induced cell apoptosis (Fig. 2C and D). The transfection of HUVECs with negative control siRNA exerted no effect on TL1A expression or cell apoptosis (Fig. 2). These findings demonstrated that VEGF is protective against high glucose-induced HUVEC apoptosis and that high glucose-induced TL1A expression promotes HUVEC apoptosis.

TL1A and VEGF are mutually inhibited in hyperglycemia. A previous study indicated that VEGF expressed in cancer cells is responsible for the downregulation of TL1A in ovarian cancer (17). In order to investigate the correlation between TL1A and VEGF expression in response to high glucose, HUVECs were transfected with TL1A siRNA or VEGF DNA and cultured in high glucose. It was demonstrated that high glucose-induced TL1A expression was inhibited by VEGF overexpression (Fig. 3A and B). By contrast, the HUVECs transfected with TL1A siRNA (50 $\mathrm{nM})$ displayed increased VEGF expression at the mRNA and protein levels compared with the control cells (Fig. 3C and D). Thus, high glucose-induced TL1A expression further inhibits VEGF expression, whereas the high glucose-induced downregulation of VEGF production results in a weakened inhibitory effect of VEGF on TL1A accumulation, resulting in elevated TL1A expression and reduced VEGF production in HUVECs in response to high glucose.

Enhanced TL1A expression and reduced VEGF production accelerate HUVEC apoptosis by attenuating the PI3K/Akt/eNOS signaling pathway. The PI3K/Akt/eNOS signaling pathway has been demonstrated to act as an important component of the mechanism that protects the cardiovasculature from endothelial cell injury. The attenuation of the $\mathrm{PI} 3 \mathrm{~K} / \mathrm{Akt} / \mathrm{eNOS}$ signaling pathway is associated with high glucose-induced HUVEC apoptosis (21). It was hypothesized that high glucose induces TL1A expression and reduces VEGF expression, thereby increasing HUVEC apoptosis by attenuating the PI3K/Akt/eNOS pathway. To test this hypothesis, HUVECs were transfected with TL1A siRNA or VEGF DNA and were cultured in high glucose for different periods. Then, $\mathrm{PI} 3 \mathrm{~K} / \mathrm{Akt} / \mathrm{eNOS}$ expression was assessed via western blot analysis. As shown in Fig. 4A, the phosphorylation of PI3K, Akt and eNOS was enhanced in cells transfected with TL1A siRNA compared with those transfected with negative control siRNA. Identical results were obtained for the HUVECs transfected with VEGF DNA to those for the HUVEC transfected with TL1A siRNA. Based on the above results, it was concluded that VEGF protects against high glucose-induced HUVEC apoptosis by activating the PI3K/Akt/eNOS pathway but that TL1A promotes HUVEC apoptosis in response to high glucose by attenuating the PI3K/Akt/eNOS pathway.

\section{Discussion}

A large body of evidence suggests that endothelial cell injury and apoptosis are likely responsible for diabetes-related cardiovascular complications. The balance between VEGF and TL1A, a pair of endogenous angiogenesis effectors that perform opposing functions, is critical for maintaining vascular integrity and homeostasis (22). The disruption of the balance between VEGF and TL1A promotes the onset of various types of disease. This study provided a comprehensive analysis of the correlation between high glucose-induced HUVEC apoptosis and the imbalance of VEGF and TL1A expression, and preliminary results demonstrating the mutual inhibitory effects between VEGF and TL1A under high glucose conditions were obtained.

It has been widely accepted that apoptosis and senescence are considered as important processes that contribute to vascular dysfunction and pathology. Previous studies have demonstrated that VEGF may aid in the survival and proliferation of endothelial cells and in the protection of cells from apoptosis by inducing the expression of anti-apoptotic and antioxidant proteins $(23,24)$. Furthermore, adequate VEGF levels are required for the regeneration and repair of endothelial cells (18). This concept was concomitant with our findings that high glucose induced the inhibition of VEGF expression, resulting in increased cell apoptosis compared with normal glucose levels. Alternatively, the overexpression of VEGF largely ameliorated high glucose-induced HUVEC apoptosis. By contrast, TL1A has been demonstrated to arrest the growth of quiescent endothelial cells and to induce the apoptosis of proliferating endothelial cells (25). Mück et al (26) reported that the overexpression of TL1A triggers premature senescence in HUVECs and that the silencing of TL1A partially alleviated HUVEC senescence. In the present study, the exposure of HUVECs to high glucose induced an increase in the apoptosis rate and the TL1A levels compared with the control. Further investigation revealed that the apoptosis of TL1A-silenced HUVECs was significantly reduced compared with that of the control cells. Based on these findings, it was proposed that decreased anti-apoptotic VEGF expression and pro-apoptotic TL1A accumulation in hyperglycemia is detrimental to survival and proliferation, finally leading to the high glucose-induced apoptosis in endothelial cells.

The above observations indicated that the disrupted balance between VEGF and TL1A results in increased HUVEC apoptosis. It has been widely accepted that maintaining the balance between TL1A and VEGF expression depends on various mechanisms, including their mutual effects. Deng et al (17) reported that HUVECs cultured in OVCAR-3 conditioned medium containing various concentrations of recombinant human VEGF exhibited significantly downregulated TL1A levels. Consistently, based on the present study, HUVECs exposed to high glucose in which VEGF was overexpressed via transfection with VEGF DNA also exhibited reduced TL1A levels, whereas VEGF expression was increased following the inhibition of TL1A expression. In addition, TL1A regulates the relative levels of two different isoforms of the VEGF receptor 1, membrane-bound (mFlt1) and soluble (sFlt1) VEGF receptor 1; and the VEGF receptor 1 is capable of inducing pro-angiogenic mFlt1 degradation and anti-angiogenic sFlt1 accumulation (27). Thus, TL1A and VEGF exert mutual inhibitory effects under high glucose conditions, and these effects may ensure the maintenance of the balance between VEGF and TL1A expression. It was speculated that high glucose-induced TL1A expression may be partially responsible for the reduction in VEGF expression. Furthermore, cell survival may be enhanced by increasing 
VEGF expression via the alleviation of high glucose-induced TL1A expression, thereby attenuating the inhibition of VEGF.

A previous study demonstrated that high glucose-induced apoptosis of human vascular endothelial cells is mediated by the activation of reactive oxygen species (ROS)-dependent c-Jun N-terminal protein kinase (JNK) and caspase-3 (28), and is prevented by the PI3K/Akt/eNOS pathway (21). It was demonstrated that the high glucose-induced increase in TL1A expression and reduction in VEGF expression promotes HUVEC apoptosis. Thus, to investigate whether the pro-apoptotic effect of enhanced TL1A expression and reduced VEGF expression is associated with the apoptotic and survival pathways described above, the activation of the PI3K/Akt/eNOS pathway was detected. HUVECs under high glucose stimulation displayed decreased VEGF expression and increased TL1A production accompanied by attenuated PI3K/Akt/eNOS pathway activation. Further investigation revealed that the PI3K/Akt/eNOS pathway was activated following the silencing of TL1A expression or the overexpression of VEGF; this activation was concomitant with decreased HUVEC apoptosis. The above results indicate that VEGF may protect against high glucose-induced endothelial cell apoptosis by activating the PI3K/Akt/eNOS pathway and that the attenuation of the PI3K/Akt/eNOS pathway may have pathological relevance to TL1A-induced apoptosis.

In conclusion, this study demonstrated that decreased VEGF levels and increased TL1A expression promote the apoptosis of HUVECs in response to high glucose conditions. Moreover, the activities of VEGF and TL1A are mutually inhibited. As a result, to prevent the occurrence and development of high glucose-induced endothelial cell injury and apoptosis, methods targeted to restore the balance between TL1A and VEGF expression by suppressing TL1A expression and enhancing VEGF production may be effective. Although the present observations strongly support the concept that TL1A is important in high glucose-induced cell apoptosis and although TL1A has been demonstrated to exert its pro-apoptotic effects in endothelial cells under high glucose conditions via multiple DR3-related signaling pathways, such as the activation of ROS-dependent JNK and caspase-3 (29), the relevance of this downstream pathway to pro-apoptosis pathways, such as the nuclear factor- $\kappa \mathrm{B}$ pathway remains to be fully elucidated. Further studies to determine the mechanisms underlying the maintenance of the balance between the anti-apoptotic effector VEGF and the pro-apoptotic effector TL1A are required.

\section{Acknowledgements}

This study was supported by the Science and Technology Plan Project of Guangdong Province (grant no. 2010B080701043). The authors would like to thank Mr. Wendong Fan et al for their technical help and Miss Ying Chen for assistance with writing.

\section{References}

1. Minamino T, Miyauchi H, Yoshida T, Ishida Y, Yoshida H and Komuro I: Endothelial cell senescence in human atherosclerosis: Role of telomere in endothelial dysfunction. Circulation 105: 1541-1544, 2002.
2. Chen J and Goligorsky MS: Premature senescence of endothelial cells: Methusaleh's dilemma. Am J Physiol Heart Circ Physiol 290: H1729-H1739, 2006.

3. Chou E, Suzuma I, Way KJ, Opland D, Clermont AC, Naruse K, Suzuma K, Bowling NL, Vlahos CJ, Aiello LP and King GL: Decreased cardiac expression of vascular endothelial growth factor and its receptors in insulin-resistant and diabetic states: A possible explanation for impaired collateral formation in cardiac tissue. Circulation 105: 373-379, 2002.

4. Waltenberger J, Lange J and Kranz A: Vascular endothelial growth factor-A-induced chemotaxis of monocytes is attenuated in patients with diabetes mellitus: A potential predictor for the individual capacity to develop collaterals. Circulation 102: 185-190, 2000.

5. Yoon YS, Uchida S, Masuo O, Cejna M, Park JS, Gwon HC, Kirchmair R, Bahlman F, Walter D, Curry C, et al: Progressive attenuation of myocardial vascular endothelial growth factor expression is a seminal event in diabetic cardiomyopathy: Restoration of microvascular homeostasis and recovery of cardiac function in diabetic cardiomyopathy after replenishment of local vascular endothelial growth factor. Circulation 111: 2073-2085, 2005.

6. Abaci A, Oğuzhan A, Kahraman S, Eryol NK, Unal S, Arinç H and Ergin A: Effect of diabetes mellitus on formation of coronary collateral vessels. Circulation 99: 2239-2242, 1999.

7. Rivard A, Silver M, Chen D, Kearney M, Magner M, Annex B, Peters K and Isner JM: Rescue of diabetes-related impairment of angiogenesis by intramuscular gene therapy with adeno-VEGF. Am J Pathol 154: 355-363, 1999.

8. Zhai YF, Ni J, Jiang GW, Lu J, Xing L, Lincoln C, Carter KC, Janat F, Kozak D, Xu S, et al: VEGI, a novel cytokine of the tumor necrosis factor family, is an angiogenesis inhibitor that suppresses the growth of colon carcinomas in vivo. Faseb J 13: 181-189, 1999.

9. Chew LJ, Pan H, Yu J, Tian S, Huang WQ, Zhang JY, Pang S and Li LY: A novel secreted splice variant of vascular endothelial cell growth inhibitor. FASEB J 16: 742-744, 2002.

10. Migone TS, Zhang J, Luo X, Zhuang L, Chen C, Hu B, Hong JS, Perry JW, Chen SF, Zhou JX, et al: TL1A is a TNF-like ligand for DR3 and TR6/DcR3 and functions as a T cell costimulator. Immunity 16: 479-492, 2002.

11. Meylan F, Davidson TS, Kahle E, Kinder M, Acharya K, Jankovic D, Bundoc V, Hodges M, Shevach EM, Keane-Myers A et al: The TNF-family receptor DR3 is essential for diverse T cell-mediated inflammatory diseases. Immunity 29: 79-89, 2008.

12. Bamias G, Martin C III, Marini M, Hoang S, Mishina M, Ross WG, Sachedina MA, Friel CM, Mize J, Bickston SJ, et al: Expression, localization and functional activity of TL1A, a novel Th1-polarizing cytokine in inflammatory bowel disease. J Immunol 171: 4868-4874, 2003.

13. Kang YJ, Kim WJ, Bae HU, Kim DI, Park YB, Park JE, Kwon BS and Lee WH: Involvement of TL1A and DR3 in induction of pro-inflammatory cytokines and matrix metalloproteinase-9 in atherogenesis. Cytokine 29: 229-235, 2005.

14. Kim WJ, Kang YJ, Suk K, Park JE, Kwon BS and Lee WH: Comparative analysis of the expression patterns of various TNFSF/TNFRSF in atherosclerotic plaques. Immunol Invest 37: 359-373, 2008.

15. Fang L, Adkins B, Deyev V and Podack ER: Essential role of TNF receptor superfamily 25 (TNFRSF25) in the development of allergic lung inflammation. J Exp Med 205: 1037-1048, 2008.

16. Hampel B, Fortschegger K, Ressler S, Chang MW, Unterluggauer H, Breitwieser A, Sommergruber W, Fitzky B, Lepperdinger G, Jansen-Dürr P, et al: Increased expression of extracellular proteins as a hallmark of human endothelial cell in vitro senescence. Exp Gerontol 41: 474-481, 2006.

17. Deng WM, Gu X, Lu Y, Gu C, Zheng Y, Zhang Z, Chen L, Yao Z and Li LY: Down-modulation of TNFSF15 in ovarian cancer by VEGF and MCP-1 is a pre-requisite for tumor neovascularization. Angiogenesis 15: 71-85, 2012.

18. Parr C, Gan CH, Watkins G and Jiang WG: Reduced vascular endothelial growth inhibitor (VEGI) expression is associated with poor prognosis in breast cancer patients. Angiogenesis 9: 73-81, 2006.

19. Conway KP, Price P, Harding KG and Jiang WG: The role of vascular endothelial growth inhibitor in wound healing. Int Wound J 4: 55-64, 2007.

20. Livak KJ and Schmittgen TD: Analysis of relative gene expression data using real-time quantitative PCR and the 2(-Delta Delta C(T)) Method. Methods 25: 402-408, 2001. 
21. Ho FM, Lin WW, Chen BC, Chao CM, Yang CR, Lin LY, Lai CC, Liu SH and Liau CS: High glucose-induced apoptosis in human vascular endothelial cells is mediated through NF-kappa $\mathrm{B}$ and c-Jun NH2-terminal kinase pathway and prevented by PI3K/Akt/eNOS pathway. Cell Signal 18: 391-399, 2006.

22. Folkman J, Watson K, Ingber D and Hanahan D: Induction of angiogenesis during the transition from hyperplasia to neoplasia. Nature 339: 58-61, 1989.

23. Zhang F, Tang Z, Hou X, Lennartsson J, Li Y, Koch AW, Scotney P, Lee C, Arjunan P, Dong L, et al: VEGF-B is dispensable for blood vessel growth but critical for their survival and VEGF-B targeting inhibits pathological angiogenesis. P Natl Acad Sci USA 106: 6152-6157, 2009.

24. Abid MR, Schoots IG, Spokes KC, Wu SQ, Mawhinney C and Aird WC: Vascular endothelial growth factor-mediated induction of manganese superoxide dismutase occurs through redox-dependent regulation of forkhead and IkappaB/NF-kappaB. J Biol Chem 279: 44030-44038, 2004.

25. Yu JY, Tian S, Metheny-Barlow L, Chew LJ, Hayes AJ, Pan H, Yu GL and Li LY: Modulation of endothelial cell growth arrest and apoptosis by vascular endothelial growth inhibitor. Circ Res 89: 1161-1167, 2001.
26. Mück C, Herndler-Brandstetter D, Micutkova L, Grubeck-Loebenstein B and Jansen-Dürr P: Two functionally distinct isoforms of TL1A (TNFSF15) generated by differential ectodomain shedding. J Gerontol A Biol Sci Med Sci 65: 1165-1180, 2010.

27. Qi JW, Qin TT, Xu LX, Zhang K, Yang GL, Li J, Xiao HY, Zhang ZS and Li LY: TNFSF15 inhibits vasculogenesis by regulating relative levels of membrane-bound and soluble isoforms of VEGF receptor 1. P Natl Acad Sci USA 110: 13863-13868, 2013.

28. Ho FM, Liu SH, Liau CS, Huang PJ and Lin-Shiau SY: High glucose-induced apoptosis in human endothelial cells is mediated by sequential activations of c-Jun NH2-terminal kinase and caspase-3. Circulation 101: 2618-2624, 2000.

29. Yue TL, Ni J, Romanic AM, et al: TL1, a novel tumor necrosis factor-like cytokine, induces apoptosis in endothelial cells-Involvement of activation of stress protein kinases (stress-activated protein kinase and p38 mitogen-activated protein kinase) and caspase-3-like protease. J Biol Chem 274: 1479-1486, 1999. 